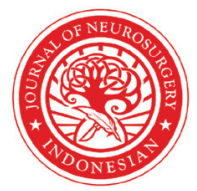

\section{Parietal giant cell glioblastoma with IDH1 mutation: A case report}

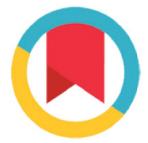

CrossMark

Indonesian Journal of Neurosurgery

\author{
Hendrikus Masang Ban Bolly ${ }^{1,2 *}$, Achmad Adam ${ }^{1}$, Hasrayati Agustina ${ }^{3}$, \\ Ahmad Faried ${ }^{1}$, Muhammad Zafrullah Arifin ${ }^{1}$
}

'Department of Neurosurgery, Faculty of Medicine, Universitas Padjadjaran - Dr. Hasan Sadikin Hospital, Bandung, West Java, Indonesia.

${ }^{2}$ Department of Biochemistry, Faculty of Medicine, Universitas Cenderawasih, Jayapura, Papua, Indonesia.

${ }^{3}$ Department of Anatomical Pathology,

Faculty of Medicine, Universitas

Padjadjaran - Dr. Hasan Sadikin

Hospital, Bandung, West Java, Indonesia.

*Corresponding to:

Hendrikus Masang Ban Bolly;

Department of Neurosurgery, Faculty of Medicine, Universitas Padjadjaran - Dr. Hasan Sadikin Hospital, Jl. Pasteur No. 38 Bandung 40161, West Java, Indonesia. Email: hendrikusbolly@gmail.com

Received: 2020-08-07

Accepted: 2021-03-13

Published: 2021-08-01

\section{ABSTRACT}

Background: Giant cell glioblastoma (GCG) is a primary glial tumor of the central nervous system. It accounts for $<1 \%$ of all glioblastoma and known as one of rare glioblastoma. It is correspondence a similar clinical feature to IDH-wildtype glioblastoma. We present a case with the diagnosis of GCG following tumor resection and histopathology examination using specific immunohistochemistry of IDH1 mutant staining.

Case presentation: A 43-year-old male with progressive headache and left extremity hemiparesis. MRI with gadolinium contrast showed a mass at the right parieto-occipital lobes with the characteristic of iso-hyperintense signal on T1W sequence, central necrosis and enhancement of the gadolinium contrast. The T2W sequence showed a hyperintense signal in the mass. Craniotomy tumor removal was performed with prone position and total removal was achieved. Histopathology finding and the immunohistochemistry staining showed results of Glial Fibrillary Acid Protein (GFAP) positive, highly proliferation index which $>10 \%$ of Ki-67 staining and positive for IDH1-R132H mutant staining. The patient survived for 38 months since the tumor resection surgery and continuing the treatment with radiotherapy and chemotherapy.

Conclusion: The role of the present therapy or IDH1 mutation status of the patient or both of them in prolonged survival time still has to be elucidated and remained a mystery.

Keywords: giant cell glioblastoma, isocitrate dehydrogenase, mutation

Cite This Article: Bolly, H.M.B., Adam, A., Agustina, H., Faried, A., Arifin, M.Z. 2021. Parietal giant cell glioblastoma with IDH1 mutation: A case report. Indonesian Journal of Neurosurgery 4(2): 56-58. D0I: 10.15562/ijn.v4i1.137

\section{INTRODUCTION}

Giant cell glioblastoma (GCG) is a rare case which counts less than $1 \%$ of all glioblastoma. ${ }^{1,2}$ This kind of glioblastoma has a better prognosis than other glioblastoma although most of them have a poor prognosis. GCG sometimes has to be differentiated with metastasis lesion in neuroimaging examinations, whether computed tomography (CT) or magnetic resonance imaging (MRI). The modality of histopathology examination and even the molecular entity of IDH1 status will contribute to a better prognostic prediction of the patient. The five years survival rate is $>10 \%$ which better than another glioblastoma (3.4\%). ${ }^{1,3,4}$ In this case report, we present a case with the diagnosis of GCG following tumor resection and histopathology examination using specific immunohistochemistry of IDH1 mutant staining.

\section{CASE PRESENTATION}

A 43-year-old male complained of a severe headache 4 days before admission. The complaint of progressive intermittent headache was lasting for 3 weeks and followed by the weakness of his left extremities. There was no history of previous stroke and other complain. Neurological examination showed a left central type cranial nerve VII paralysis.

The results of routine blood examinations were normal. MRI with gadolinium contrast showed a mass at the right parieto-occipital lobes with the characteristic of iso-hyperintense signal on T1W sequence, central necrosis and enhancement of the gadolinium contrast. The T2W sequence showed a hyperintense signal in the mass. There were peritumoral edema and midline were shifting to the contralateral direction of the mass. There was a global compression of the sulci and gyri (Figure 1).

The work up diagnosis was suspected of a glioma. Craniotomy tumor removal was performed with prone position and total removal was achieved. The tumor tissue shows a macroscopic characteristic of a hypervascularized mass. Some parts of the tumor were easy to aspirate by the suction cannula and some parts were yellowish tissue. The solid components were collected for further histopathology examinations. A full decompression was achieved and the bleeding was controlled.

We examined multiple sections and staining for microscopic examination. Multinucleated giant cells and small fusiform cell were specific histologic characteristic found microscopically. Some giant cells with palisading and large ischemic necrosis were observed. A specific formation of a pseudo rosette-like pattern with a typical feature of mitotic was also noted. Glial Fibrillary Acid Protein (GFAP) examination showed positive results combine with a high index (> 10\%) of proliferation confirmed 
with Ki-67 staining. Interestingly, our immunohistochemistry examination of IDH1 R132H mutant showed positive expression of the mutations (Figure 2). The final results of histopathology conclude as giant cell glioblastoma with IDH1 mutation-positive.
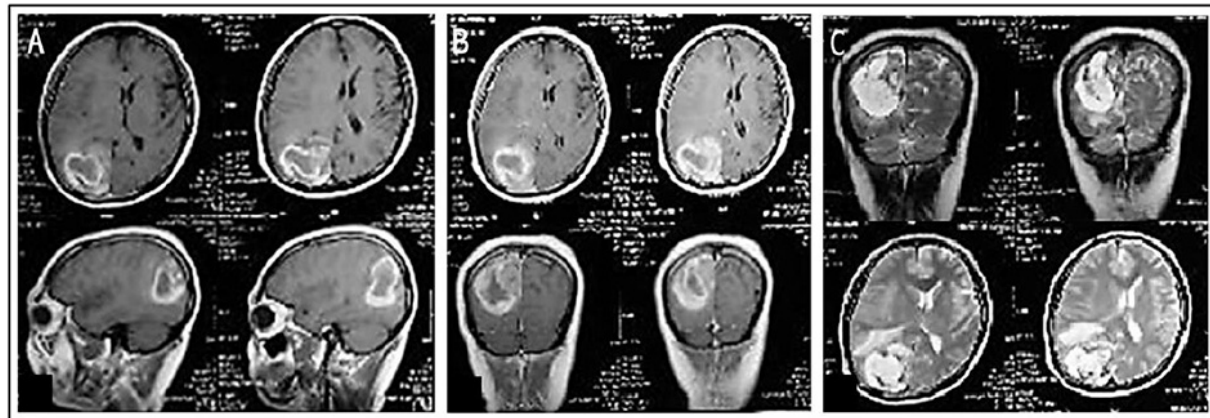

Figure 1. MRI of the patient. A. T1W axial (above) and sagittal (below) showing a hypointense signal in central of the tumor. B. T1W with gadolinium contrast axial (above) and coronal (below) showing a hypointense signal in the central of the tumor and ring enhancement in the tumor. C. T2W coronal (above) and axial (below) showing a hyperintense signal.

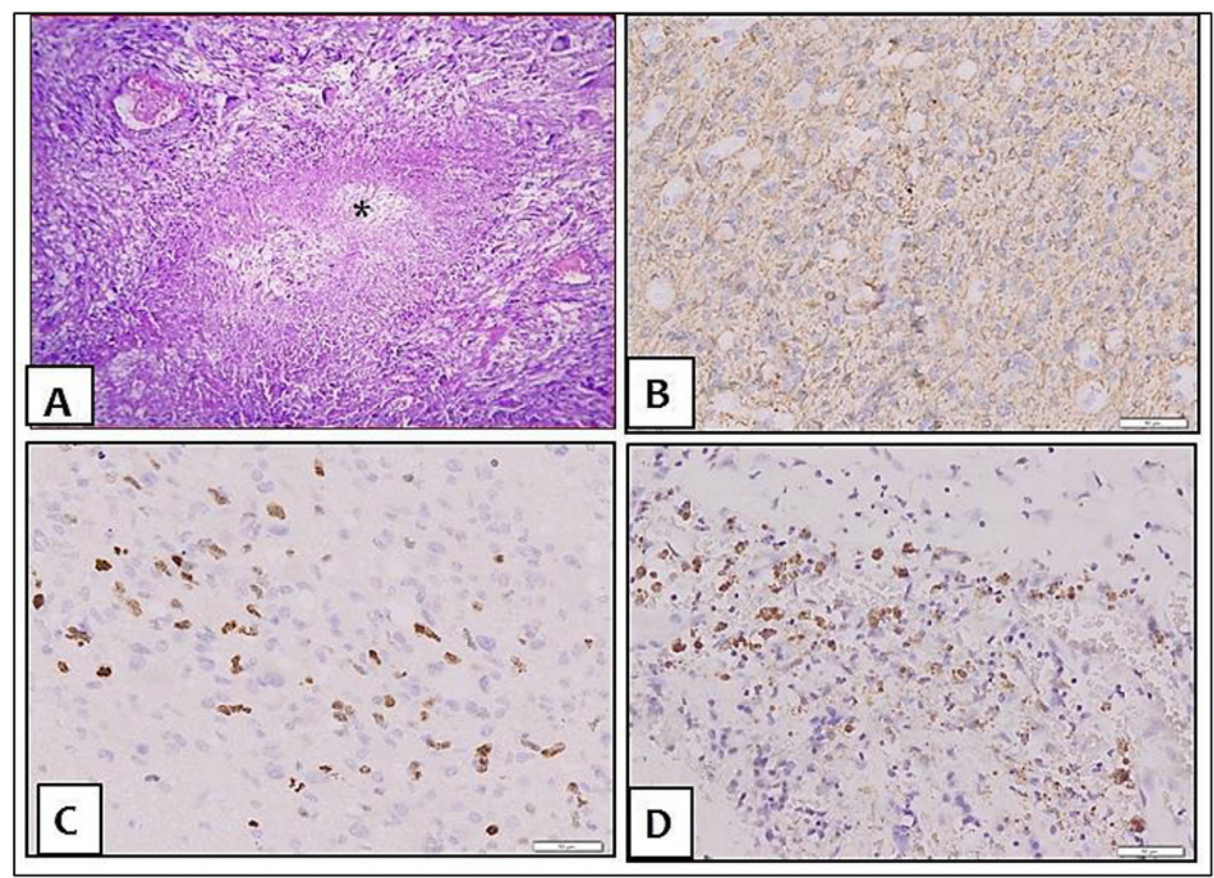

Figure 2. A. HE staining showed a bizarre appearing giant cells, multinucleated eosinophilic dominant, extensive necrosis $\left(^{*}\right)$ and smaller fusiform cells (H\&E, 100x). B. IHC staining of glial fibrillary acidic protein (GFAP) [100x]: indicate the origin of the tumor was a glial cell. C. IHC staining of Ki-67 [200x]: showed a high index $(>10 \%)$ proliferation indicate a high-grade glioma characteristic. D. IHC staining of mutant IDH1 R132H [200x]; showed an immunoreactive results, indicate a specific mutation of isocitrate dehydrogenase I gene at codon 132 of amino acid, changing of Arginine (R) to Histidine $(\mathrm{H})$. a grade IV from astrocytic origins. ${ }^{3}$ Histology appearance shows a highly cellular of the specific size of the tumor cells $(500 \mu \mathrm{m})$. There is pseudo palisading necrosis or a large ischemic form. The giant cells also show lipid accumulation and microcalcifications. ${ }^{3}$

The manifestation of GCG was similar to classic glioblastoma, which depends on the tumor location. GCG predominantly developed in subcortical white matter in the temporal and parietal lobes., ${ }^{1,46}$ The other location also found in the cerebellum, lateral ventricles, optic chiasm, and spinal cord. ${ }^{3}$ Turner et al. concluded that the radiologic appearance of the GCG is not well documented. Otherwise, the characteristic consideration could be the same as a classic radiologic feature of glioblastoma. ${ }^{4}$

The main characteristic of the symptoms develops as focal neurological deficit and increase of the intracranial pressure due to peritumoral edema. ${ }^{5}$ Our patient has the symptoms of progressive headache and paresis of the cranial nerve VII followed by left hemiparesis. The neuroimaging of glioblastoma shows an irregular shape with ring enhancement and central necrosis. MRI shows hypointense signal on T1W and hyperintense on T2W that surrounded by edema. ${ }^{1,3,5}$ The specific mural nodule surrounds the cystic parts has been signed in the MRI. ${ }^{3}$

Interestingly, in this case, there was IDH1 mutation (Fig. 2D). Theoretically, GCG do not express IDH mutations and consider as the variant of IDHwildtype glioblastoma. However, the literature showed that there was $5 \%$ of the GCG with IDH1/2 mutations. ${ }^{1,5}$ The IDH1/2 mutations occurred $100 \%$ in secondary glioblastoma (IDH mutant) and $0 \%$ reported in both of primary glioblastoma and gliosarcoma. ${ }^{1}$ The peak ages of the GCG diagnosis vary from 42 to 44 years old. The average ages were 54.5 years old and predominant in males (the male-to-female ratio is 1.6). ${ }^{1,3,5}$ It was similar to our patients as a male and 43 years old. GCG could be associated with neurofibromatosis type 1 and tuberous sclerosis. ${ }^{3}$

The molecular characteristics of the GCG are PTEN mutation (33\%), ATRX expression loss 
(19\%), TERT mutation (25\%), and high frequency of TP53 mutation (84\%). ${ }^{1}$ TP53 can be detected by immunohistochemistry staining because of stable mutant protein. ${ }^{3}$ PTEN gene related to cell cycle arrest, apoptosis and inhibition of cell motility. Mutation of this gene allows the cell to start cell division. Another abnormality that found in GCG is the abnormality of the chromosome (loss of 17p, 1p, 19q). ${ }^{1,3}$ In this case, we had examined for a specific IDH1 R132H mutant and found positive results. These mutations contribute to the prolonged survival rate of the patients. We followed up the patients 38 months after the surgery and the patient was still in good condition. USA National Cancer Database found the overall survival of the patient was 13.5 months. ${ }^{1}$

The treatment strategy for GCG included surgery, radiotherapy, and chemotherapy. ${ }^{3,6}$ These modalities contribute to prolong mean survival time up to 8 months. ${ }^{3}$ The combination of radiation and chemotherapy (Temozolomide) after the surgery were associated with delayed tumor progression. ${ }^{2}$

We proposed that, the IDH1 mutations perhaps have the role of the longer survival time of the patients. This finding has to be proven by a further large sample study. IDH1 mutation was the evidence that the GCG was established gradually from grade II (diffuse astrocytoma) to become grade III (anaplastic astrocytoma) and then grade IV (glioblastoma) at the end. ${ }^{6,7}$ There are $50-75 \%$ of grade II that develop to grade IV of glioblastoma. ${ }^{8}$ We use a specific antibody of IDH1 R132H mutant to detect the IDH1 mutation. This codon of Arginine-132 was a "hot spot" location or the mutations in the IDH1 gene. The missense mutations change (395:G à A) and resulted in the alteration of Arginine to Histidine. The translations affect the normal function of isocitrate dehydrogenase- 1 which catalyzes the isocitrate to $a$-ketoglutarate change to become D-2-hydroxyglutarate.
The D-2-hydroxyglutarate was an oncometabolite that has a dominant role in tumorigenesis. ${ }^{6,7,9,10}$ Epidemiology studies showed that the mutations make the survival time become longer. ${ }^{8,11,12}$ Therefore, mutations of IDH1 contribute to the prolonged survival time of the GCG patients.

\section{CONCLUSION}

The diagnosis of rare GCG was presented with post tumor resection, histopathology examination and molecular examination of IDH1 mutant status. The patient received additional chemo-radiotherapy and showed a prolonged survival time than the average. The role of the present therapy or IDH1 mutation status of the patient or both of them in prolonged survival time still has to be elucidated and remained a mystery.

\section{CONFLICT OF INTEREST}

Author declares no conflict of interest regarding this study.

\section{AUTHOR CONTRIBUTION}

Author contribute equally in this study.

\section{FUNDING}

Author declare no sponsorship regarding this study.

\section{REFERENCES:}

1. Ohgaki H, Klehues P, Plate KH, Nakazato Y, Bigner DD. Giant cell glioblastoma. In: Louis DN, et al. (eds.) WHO Classification of Tumours of the Central Nervous System Revised 4th Ed. France: International Agency for Research on Cancer; 2016. p. 46 - 47.

2. Oh T, Rutkowski MJ, Safaee M, Sun MZ, Sayegh ET, Bloch O, et al. Survival outcomes of giant cell glioblastoma: Institutional experience in the management of 20 patients. J Clin Neurosci. 2014; 21(12): 2129 - 34. https://doi. org/10.1016/j.jocn.2014.04.011.

3. Valle-Folgueral JM, Mascarenhas L, Costa JA, Vieira F, Soares-Fernandes J, Beleza P, Alegria C. Giant cell glioblastoma: Review of the literature and illustrated case. Neurocirugia. 2008;19(4): 343 - 9. https://doi.org/10.1016/ s1130-1473(08)70221-5.

4. Turner R, Matthys S, Heymann J, Gelman B. Imaging findings in the progression of giant cell glioblastoma. Radiol Case Rep. 2018;13(5): 1007 - 11. https://doi.org/10.1016/i. radcr.2018.07.010.

5. Louis DN, Suva ML, Burger PC, Perry A, Kleihues $\mathrm{P}$, Aldape KB, et al. Glioblastoma IDH-wildtype. In: Louis DN, et al. (eds.) WHO Classification of Tumours of the Central Nervous System Revised 4th Ed. France: International Agency for Research on Cancer; 2016. p. 28 45.

6. Louis DN. WHO classification and grading of tumours of the central nervous system. In: Louis DN, et al. (eds.) WHO Classification of Tumours of the Central Nervous System. France: International Agency for Research on Cancer; 2016. p.12 - 13 .

7. Mellai M, Piazzi A, Caldera V, Monzeglio O, Cassoni P, Valente G, Schiffer D. IDH1 and IDH2 mutations, immunohistochemistry and associations in a series of brain tumors. $J$ Neurooncol. 2011; 105(2): 345 - 357. https://doi. org/10.1007/s11060-011-0596-3.

8. Nunez FJ, Mendez FM, Kadiyala P, Alghamri MS, Savelieff MG, Garcia-Fabiani MB, et al. IDH1-R132H acts as a tumor suppressor in glioma via epigenetic up-regulation of the DNA damage response. Sci Transl Med. 2019, 11(479): eaaq1427. https://doi.org/10.1126/ scitranslmed.aaq1427.

9. Ohba S, Hirose Y. Biological significance of mutant isocitrate dehydrogenase 1 and 2 in gliomagenesis. Neurol Med Chir. 2016; 56(4): 170 - 179. https://doi.org/10.2176/nmc. ra.2015-0322.

10. Waitkus MS, Diplas BH, Yan $\mathrm{H}$. Isocitrate dehydrogenase mutations in gliomas. Neuro Oncol. 2016; 18(1): 16 - 26. https://doi. org/10.1093/neuonc/nov136.

11. Tao QS, Lei Y, Si G, YanQing D, HuiXia H, XueLin Z, et al. IDH mutations predict longer survival and response to temozolomide in secondary glioblastoma. Cancer Sci. 2012; 103(2): 269 - 273. https://doi.org/10.1111/ j.1349-7006.2011.02134.x.

12. Okita Y, Narita Y, Miyakita Y, Ohno M, Matsushita Y, Fukushima M, et al. IDH1/2 mutation is a prognostic marker for survival and predicts response to chemotherapy for grade II gliomas concomitantly treated with radiation therapy. Int J Oncol. 2012; 41(4): 1325 - 36. https://doi.org.10.3892/ijo.2012.1564.

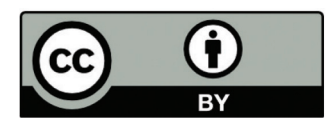

This work is licensed under a Creative Commons Attribution 